\title{
Effects of Visitor Presence and Crowd Size on Zoo-Housed Red Kangaroos (Macropus Rufus) During and After a COVID-19 Closure
}

\author{
Megan Jones*, Kylen N. Gartland, and Grace Fuller \\ Center for Zoo and Aquarium Animal Welfare and Ethics, Detroit Zoological Society \\ *Corresponding author (Email: mjones@ dzs.org)
}

Citation - Jones, M., Gartland, K. N., \& Fuller, G. (2021). Effects of visitor presence and crowd size on zoo-housed red kangaroos (Macropus rufus) during and after a COVID-19 closure. Animal Behavior and Cognition, 8(4), 521537. https://doi.org/10.26451/abc.08.04.06.2021

\begin{abstract}
Macropods, particularly kangaroos and wallabies, are common species included in walk-through habitats that put them in close proximity to zoo visitors. However, there has been little research into how visitor presence and density impact the welfare of these individuals. We monitored the behavior and space use of fifteen red kangaroos (Macropus rufus) for a total of ten weeks during and after a nearly three-month zoo closure due to the COVID-19 pandemic. Our findings revealed potential visitor effects, evidenced by more time spent in social proximity, greater inactivity, and more restricted space use after the zoo reopened. Although temperature and weather likely played a role in at least some of these behavioral changes, social proximity and space use changed with crowd size in a manner consistent with our zoo status (i.e., open or closed) results. Additionally, time spent feeding was significantly related to crowd size but not zoo status, such that the kangaroos spent more time feeding when there were no visitors in the habitat. These findings suggest that visitor effects explain these behavioral changes better than seasonal confounds. We also noted several individual differences in response to visitor presence and crowd size, highlighting the importance of evaluating behavioral responses to visitors on an individual basis.
\end{abstract}

Keywords - Visitor effects, Red kangaroo, Macropods, Walk-through habitat, Zoo, Welfare

The systematic evaluation of animal welfare began in the 1960s with the Five Freedoms model, which sought to ensure basic welfare standards for the poultry and livestock farming industries by minimizing negative affective states such as pain, hunger, discomfort, and fear (Webster, 1994, pp.10-14). More prevalent today is the Five Domains model, which takes into account positive affective states and outlines five categories that contribute to an animal's overall welfare state: nutrition, environmental conditions, health, behavior, and mental state (Mellor \& Beausoleil, 2015; Mellor \& Reid, 1994). A recent amendment to the Five Domains Model emphasizes human-animal interactions as a critical variable affecting animal welfare, with those interactions categorized based on whether the human subject is familiar (i.e., a caretaker) or unfamiliar (i.e., a visitor) to the animal (Mellor et al., 2020).

Hosey (2000) classified zoo animals' relationships to visitors as either a source of stress, a source of enrichment, or neutral. To date, studies of visitor effects have found evidence for all of these effects, with varying and sometimes conflicting results. An independent analysis by Boyle et al. (2020) determined that, out of 16 different species, $90 \%$ of mammals and $60 \%$ of fish had at least one behavioral change associated with visitor abundance or behavior. Visitor presence and density have been associated with increased levels of abnormal behavior or aggression across several species, including mandrills 
(Mandrillus sphinx), Diana monkeys (Cercopithecus diana), lion-tailed macaques (Macaca silenus), jaguars (Panthera onca), black-capped capuchins (Sapajus apella), and western lowland gorillas (Gorilla gorilla gorilla) (Chamove et al., 1988; Mallapur et al., 2005; Sellinger \& Ha, 2005; Sherwen, Harvey, et al., 2015; Stoinski et al., 2012; Wells, 2005). Among some felid species, the presence of visitors has also been linked to decreased activity levels, and increases in visitor density have been related to decreased time spent visible (Mallapur \& Chellam, 2002; Sellinger \& Ha, 2005; Suárez et al., 2017). Several species of birds and other mammals have also been found to display visitor avoidance behavior (Blanchett et al., 2020; Chiew et al., 2020; Sherwen, Magrath, et al., 2015; Stevens et al., 2013).

However, these results are not consistent across all studies, as some have reported no visitor impacts on behavior or space use (Bonnie et al., 2016; Margulis et al., 2003; O'Donovan et al., 1993). Lesser anteaters (Tamandua tetradactyla) and flamingos (Phoenicopterus sp.) did not show any significant changes in their activity budgets or space use in response to higher visitor numbers (Chiapero et al., 2021; Rose et al., 2018). Some individuals have also been observed to approach visitors during periods of higher density, suggesting visitors were stimulating or otherwise promoted positive affect (Eltorai \& Sussman, 2010). Additionally, Diana monkeys have also demonstrated positive welfare indicators with higher densities of visitors (Todd et al., 2007).

Visitor effects should also be considered on an individual basis, as different individuals can have varying behavioral responses to the same environmental stimuli (Boyle et al., 2020; Hashmi \& Sullivan, 2020; Kelly et al., 2015; Stoinski et al., 2012). Zoos have largely been moving towards a welfare assessment model that emphasizes the importance of individual-based monitoring and care (Kagan et al., 2015). As such, studies of visitor effects should also include a component of individual-based analysis to promote both individual and group welfare. Welfare assessments that encapsulate human-animal interactions are encouraged to consider variables such as an animal's history of prior human contact, the complexities of the human-animal bond, and physical proximity between humans and animals (Mellor et al., 2020). The latter point may be particularly critical for animals in walk-through habitats, which are common for zoo-housed red kangaroos (Macropus rufus).

Red kangaroos are the largest living marsupial (Strahan, 1983; Wilson \& Mittermeier, 2015) and a common species in zoos. They are native to Australia's deserts and grasslands and, like many grassland species, congregate together into semi-nomadic groups ranging from two to over one hundred individuals (Frith \& Calaby, 1969). Group life protects from predators (Blumstein \& Daniel, 2003; Domico, 1993; Frith \& Calaby, 1969) through increased vigilance, as kangaroos primarily rely on outrunning predators rather than hiding (Wilson \& Mittermeier, 2015). They are primarily nocturnal, spending most of their time seeking shade and intermittently sleeping throughout the day before foraging for food after dark and into the early morning (Frith \& Calaby, 1969, Wilson \& Mittermeier, 2015).

Despite the frequency with which kangaroos and other macropods are featured in walk-through habitats, the impacts of visitor presence and proximity on their behavior are relatively understudied. Koalas (Phascolarctos cinereus) have responded to higher visitor numbers and noise levels with increased vigilance (Larsen et al., 2014), and both visitor density and time of day have been found to significantly impact the behavior and space use of red-necked wallabies (Macropus rufogriseus fruticus) (Lockley \& Leadbeater, 2005). Quokkas (Setonix brachyurus) in a walk-through habitat were less visible on days when the habitat was open to visitors (Learmonth et al., 2018), and studies on kangaroos in similar habitats found that both red kangaroos and Kangaroo Island kangaroos (Macropus fuliginosus fuliginosus) spent more time performing vigilance behaviors as visitor numbers increased (Sherwen, Hemsworth, et al., 2015).

Queiroz and Young (2018) published a model that predicts mammals' responses to visitors based on several natural history characteristics, such as habitat, activity cycle, diet, and stratum use. They found that mammals from open habitats, such as grasslands, are generally less affected by visitors than species found in areas with more cover. Nocturnal mammals were less affected by visitors than diurnal species, likely because visitors tended to be present during their period of inactivity. Herbivorous and omnivorous mammals tended to be more affected by visitor presence than carnivores due to their increased likelihood of predation, and terrestrial species were more susceptible to visitor effects than arboreal species that can 
rely on vertical space to distance themselves from visitors (Queiroz \& Young, 2018). This model is consistent with many studies that have demonstrated adverse impacts of visitors on primates (Chamove et al., 1988; Mallapur et al., 2005; Sherwen et al., 2015; Stoinski et al., 2012; Wells, 2005), which are often diurnal, omnivorous, and inhabit heavily forested areas (Mittermeier et al., 2013). However, the model has shown less consistent results when compared to visitor effect studies of other mammals such as bobcats (Lynx rufus) (Suárez et al., 2017), lesser anteaters (Chiapero et al., 2021, and black-tailed prairie dogs (Cynomys ludovicianus) (Eltorai \& Sussman, 2010). Based on the model, red kangaroos would be classified as a relatively low level of concern for potential visitor effects because of their tendency to be found in open grasslands and their predisposition to sleeping during the time of day when visitors are present. However, the model suggests that their vulnerabilities as terrestrial prey animals might mean they are inclined to engage in at least some visitor avoidance behavior.

This study explored how visitor presence and crowd size potentially impacted the welfare of red kangaroos housed in a walk-through habitat by monitoring their behavior and use of space. The study was conducted during the onset of the COVID-19 pandemic when the Detroit Zoo, which is usually open 362 days of the year, was closed to guests for several months before reopening with limited attendance capacity. Our aim was to use this unprecedented opportunity to study visitor effects on an underrepresented species on a much longer time scale than is represented in the literature. Previous studies on visitor effects have used visual barriers (Chiew et al., 2020; Sherwen et al., 2015), variations in crowd size (Sellinger \& Ha, 2005; Sherwen, Hemsworth, et al., 2015), noise level (Larsen et al., 2014), visitor proximity (Chiew et al., 2019), and comparisons between open and closed days (Mallapur \& Chellam, 2002). This study builds on previous visitor effect studies on kangaroos by examining not only the animals' behavior with varying crowd sizes but also during and after a complete absence of visitors for a prolonged period. Given the unusual circumstances, we hypothesized that changes in behavior and space use would occur in response to visitor presence and crowd size, such as a reduction in space use and more time inactive in the presence of visitors and at higher crowd densities.

\section{Method}

\section{Ethical Statement}

This study involved non-invasive observational research and was exempt from review by the Detroit Zoological Society's Animal Welfare and Management Committee.

\section{Subjects and Study Site}

The subjects for this study included six male and nine female red kangaroos that resided with three red-necked wallabies (Macropus rufogriseus) in the Detroit Zoo's Australian Outback Adventure (Figure 1). All individuals were born at the Detroit Zoo except Lilly, a female born at another accredited zoological facility and transferred to the Detroit Zoo in August 2018, and Kelly, a male rescued from a private owner and placed at the zoo in August 2019. Each individual was identified using facial features and existing ear tags (Table 1). The $7,896 \mathrm{~m}^{2}$ walk-through habitat consisted primarily of grass, live trees, and a small pool. The habitat was enclosed by cyclone fencing as well as a 1.8-2.4 $\mathrm{m}$ gunite perimeter. Both the kangaroos and wallabies had unrestricted access to the habitat, as well as their primary indoor habitat space and a covered outdoor shelter. During normal operating hours, the habitat was monitored by a team of trained zoo volunteers who instructed guests to stay on the pathway and avoid touching or feeding the animals.

Animal care staff typically entered the habitat twice a day to assess the kangaroos and provide food. The kangaroos received browse, including mulberry and eucalyptus, once or twice a day in the habitat. On three days a week, they also received $1.8 \mathrm{~kg}$ chopped produce (carrots, yams, apples, and romaine) scattered in the habitat. Their daily primary diet was provided in the indoor habitat in the afternoon after the space was serviced. It consisted of .9-1.0 kg of pelleted Mazuri Macropod Low Starch 
food (\#0038665, PMI Nutrition International, LLC, St. Louis, MO, USA) per individual and 3-4 flakes of soft hay for the group. Hay was reduced to 1-2 flakes per day once grazing opportunities became more readily available in the summer. The habitat itself provided seasonal opportunities to graze on grass and other native plants.

\section{Figure 1}

Aerial View of the Australian Outback Adventure

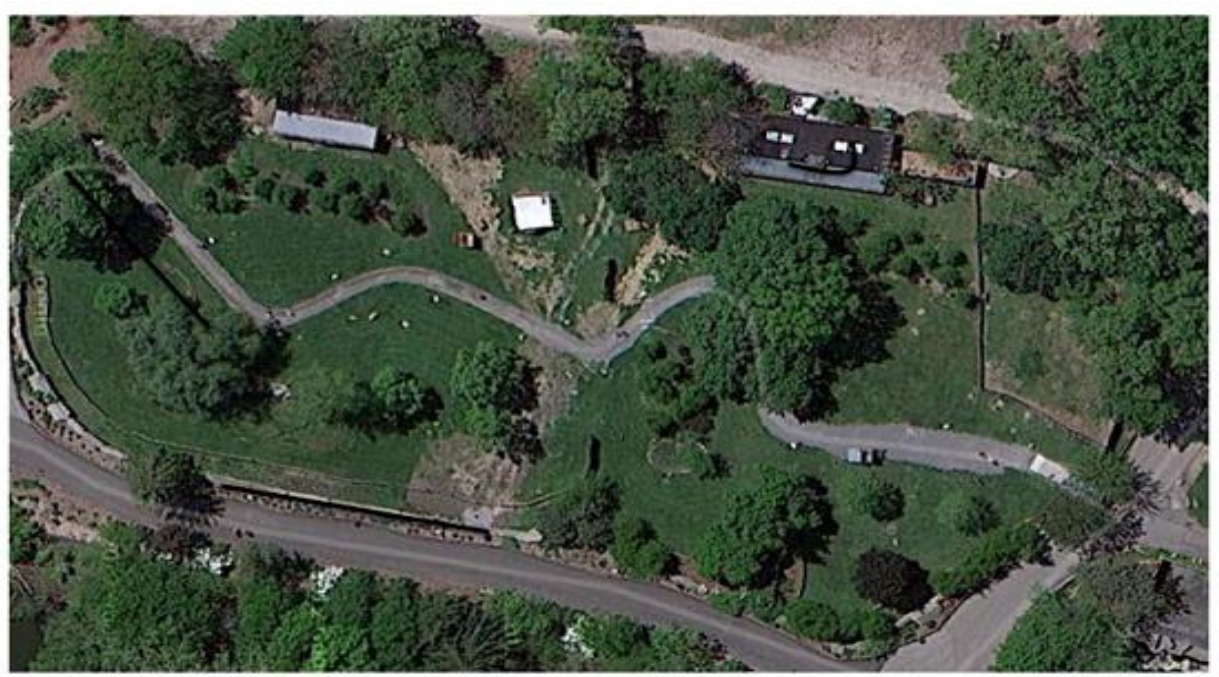

Note. Map data @2020 Google

Table 1

Names, Ages, and Sexes of Study Individuals

\begin{tabular}{ccc}
\hline Name & Sex & Age at Time of Monitoring \\
\hline Isa & Female & 9 \\
Mackay & Female & 8 \\
Stirling & Female & 8 \\
Rae & Female & 13 \\
Missy & Female & 14 \\
Marrabel & Female & 6 \\
Kiki & Female & 6 \\
Zora & Female & 7 \\
Lilly & Female & 3 \\
Boomer & Male & 8 \\
Jasper & Male & 9 \\
Hampton & Male & 9 \\
Grafton & Male & 8 \\
Logan & Male & 6 \\
Kelly & Male & 2 \\
\hline
\end{tabular}




\section{Behavioral Observations}

We conducted 125 observations totaling approximately $21 \mathrm{hrs}$ across five weeks from April 30 to June 5, 2020 when the zoo was closed due to COVID-19, and five weeks from June 10 to July 17, 2020 when the zoo reopened to the public. We collected scan samples three times a day Monday through Friday in the morning (0800-1100 hr), midday (1100-1400 hr), and afternoon (1400-1700 hr). During the reopening period, observations collected during the 0800-1100 hr time period were sometimes collected before the zoo opened for the day at $0900 \mathrm{hr}$. During each observation, we recorded every individual's behavior, location, social proximity, and proximity to the visitor path on a single interval according to a predetermined ethogram (Altmann, 1974) (Table 2). Each session lasted approximately ten minutes. Before collecting data, observers scored the temperature, weather conditions (sunny, partly cloudy, overcast, or precipitation), keeper presence, and crowd size in the habitat $(0,1-5,6-10,11-15$, or $16+$ visitors present). Two observers collected data and achieved above $90 \%$ inter-observer reliability, which we tested using the percentage of observer agreement across 3 observations. In order to avoid any potential influence on behavior, we conducted all observations outside of the habitat using binoculars and logged data via the ZooMonitor app (Ross et al., 2016).

Table 2

Ethogram of Behavior, Path Proximity, and Social Proximity

\begin{tabular}{|c|c|}
\hline Item & Definition \\
\hline \multicolumn{2}{|l|}{ Behavior } \\
\hline Social Agonistic & boxing, pushing, hitting, or chasing other kangaroos or wallabies in the habitat \\
\hline Social Affiliative & $\begin{array}{l}\text { mounting, copulating, touching another with paws, allogrooming, sniffing, or tolerating } \\
\text { sniffing }\end{array}$ \\
\hline Visitor-Directed & $\begin{array}{l}\text { touching, licking, hitting, kicking, following, or posturing at or vocalizing at visitors in the } \\
\text { habitat }\end{array}$ \\
\hline Keeper-Directed & $\begin{array}{l}\text { touching, licking, hitting, kicking, following, or posturing at or vocalizing at an animal care } \\
\text { staff person in the habitat }\end{array}$ \\
\hline Moving & movement through the habitat, including bipedal, quadrupedal, or pentapedal movement \\
\hline Feeding & $\begin{array}{l}\text { ingesting food or searching for food; includes having nose in the grass with or without jaw } \\
\text { movement or drinking }\end{array}$ \\
\hline Maintenance & self-grooming or voiding behaviors \\
\hline Inactive & stationary with eyes open or closed; can be sitting, standing bipedally, or lying down \\
\hline Other & digging or other undefined behaviors (include note) \\
\hline Not Visible & the animal or its behavior cannot be seen \\
\hline
\end{tabular}




\begin{tabular}{|c|c|}
\hline Item & Definition \\
\hline \multicolumn{2}{|l|}{ Path Proximity } \\
\hline On Path & the animal is on the visitor path \\
\hline Near Path & the animal is within one body length (not including the tail) of the path \\
\hline Distant to Path & the animal is more than one body length (not including the tail) away from the path \\
\hline Unclear/Not Visible & proximity to the path cannot be determined or the animal is not visible \\
\hline \multicolumn{2}{|l|}{ Social Proximity } \\
\hline Proximate & $\begin{array}{l}\text { the animal is within one body length (not including the tail) or in contact with a kangaroo } \\
\text { or wallaby }\end{array}$ \\
\hline Distant & $\begin{array}{l}\text { the animal is more than one body length (not including the tail) away from all other } \\
\text { kangaroos and wallabies }\end{array}$ \\
\hline Unclear/Not Visible & the animal is not visible or its proximity to others cannot be determined \\
\hline
\end{tabular}

We conducted group level analyses using the total number of complete observations. For each observation, we calculated the number of kangaroos engaged in each behavior out of the total visible kangaroos. As such, the results are presented in terms of the average percent of kangaroos per observation visibly engaging in a specified behavior with an accompanying standard error to account for variation between observations. Although we recorded social agonistic behavior, there was only one observed instance. As such, we did not include this dependent variable in analyses. Ultimately, we tested seven dependent behavioral variables: social affiliation, movement, feeding, maintenance behaviors, inactivity, path proximity, and social proximity. We first ran Kolmogorov-Smirnov tests for normality using the UNIVARIATE procedure in SAS $\mathbb{0}$, 9.4.1 (Cary, NC, USA). The data were not normally distributed, so we elected to proceed with non-parametric statistical analyses. Following previously established methodology, we ran Wilcoxon two-sample tests and corrected for small sample size through a Monte Carlo sampling method at 10,000 permutations which generated the reported test statistic (Colgrave et al., 2006; Leeds \& Lukas, 2019; Plowman, 2008). This allowed us to statistically assess variation in our dependent variables between zoo status conditions. We then used Kruskal-Wallis tests with the same Monte Carlo corrections to examine variation in our dependent variables between crowd size conditions. We then ran another Spearman correlation examining the relationships between our potentially confounding environmental variables (weather and temperature) and our dependent behavioral variables. Keepers were present for only 9 of 125 total observations. We ran duplicate analyses excluding observations when keepers were present but elected to retain the full dataset as keeper presence did not impact the results.

We were also interested in variation in habitat space use between zoo status, crowd size, and time of day conditions. In order to address this question, we used space use data to calculate an evenness index. A number of evenness indices have been proposed and utilized for the ecological study of space use (Brereton, 2020; Payne et al., 2005), including the Dispersion index. The Dispersion index, represented by $\mathrm{D}_{\mathrm{L}}$, is calculated as follows: 


$$
D_{L}=\frac{-\Sigma^{s} p i(\ln (p i))}{\ln \left(S_{s}\right)}
$$

and represents a variant of the Shannon diversity index, which is frequently used for investigating behavioral diversity under varying environmental conditions (Halliday, 1983; Payne et al., 2005; Shannon, 1948). Traditionally, $p i$ is the proportion of individuals of a species at site $i, s$ is the number of sites occupied by that species, and $S_{s}$ is the total number of sites surveyed (Payne et al., 2005). Resultant values range from 0 (sparse) to 1 (dense). This dispersion index has been adapted with slight modifications for differing space use conditions (Belton et al., 2016). For the purposes of our study, $p i$ represents the proportion of visible scans, $S$ represents the location, and $S_{s}$ represents the total number of surveyed locations. We used a total of 55 locations to calculate the Dispersion index, and the resulting number represents the proportion of total available space used by an individual in a given condition. We calculated a dispersion index for each individual for the following conditions: zoo status (open versus closed), crowd size $(0,1-5,6-10,11-15,16+)$, and time of day (morning, midday, afternoon) by zoo status. We then ran generalized linear models (GLMs) using the PROC GLM function in SAS to examine variation in habitat dispersion between zoo status and crowd size conditions. For all inferential statistics, we used a significance cutoff of $p<.05$.

Because animal welfare is measured at the individual level, we also ran descriptive statistics to examine potential behavioral variation between crowd size conditions and zoo status conditions for individual animals. Similar to the group level analyses, results at the individual level are presented in terms of the percent of time that individual spent engaged in a specific behavior averaged across the total complete observations for that individual.

\section{Results}

\section{Zoo Status}

Group means represent the percent ( \pm standard error, SE) of visible kangaroos engaged in a behavior averaged across observations, while individual means are the percent ( \pm SE) of visible time observed. In terms of zoo status, inactivity was significantly higher when the zoo was open $(82.74 \% \pm$ $2.00 \%, N=62)$ than when the zoo was closed $(75.12 \% \pm 2.56 \%, N=60)$ (Table 3) (Figure 2). When the zoo was closed, individual inactivity accounted for anywhere from $65.96 \% \pm .50 \%$ of total visible time for Hampton to $84.62 \% \pm 0.45 \%$ for Missy. When the zoo was open, individual inactivity ranged from $70.49 \pm 0.47 \%$ (Boomer) to $90.91 \% \pm 0.49 \%$ (Kelly). The trend of increased inactivity when the zoo reopened to guests was consistent across every individual except for Isa. Isa spent approximately $82.98 \%$ $\pm 0.49 \%$ of her time inactive when the zoo was closed and $78.33 \% \pm 0.45 \%$ of her time inactive when the zoo was open. In terms of potential confounds, we found that inactivity was positively correlated with temperature $(r(120)=.20, p=.02)$.

We observed a similar trend for social proximity in which individuals spent more time in proximity when the zoo was open than when the zoo was closed (Open: $80.57 \% \pm 1.78 \%, N=62$; Closed: $59.34 \pm 3.07 \%, N=58$ ) (Table 3). Excepting Missy and Kelly, individuals spent anywhere from 77.59\% $\pm .41 \%$ (Logan) to $98.36 \pm .03 \%$ (Boomer) of visible time in social proximity to another kangaroo when the zoo was open. This is in comparison to a range of $48.84 \% \pm 0.50 \%$ (Rae) to $82.00 \% \pm 0.06 \%$ (Boomer) when the zoo was closed. Of the two outlying individuals, Missy spent $41.18 \% \pm 0.50 \%$ of her time in social proximity when the zoo was closed and only $51.72 \% \pm 0.50 \%$ of her time in social proximity when the zoo was open. Kelly was even more notable in spending only $15.15 \% \pm 0.50 \%$ of his time in social proximity when the zoo was closed and $9.09 \pm .49 \%$ in social proximity when the zoo was open. Kelly was the only individual that decreased time spent in social proximity after the zoo was reopened to visitors. As a potential confound for these results, we found that social proximity was positively correlated with temperature $(r(118)=.50, p<.001)$. 
Table 3

Wilcoxon Two-Sample Tests with Monte Carlo Correction Results for Behavioral Variation Between Zoo Status Conditions

\begin{tabular}{lcccc}
\hline \multicolumn{1}{c}{ Behavior } & Statistic $(\mathrm{S})$ & $\mathrm{Z}$ & $\mathrm{Pr}>|\mathrm{Z}|$ & $\mathrm{Pr}>|\mathrm{S}-\mathrm{Mean}|$ \\
\hline Social Affiliation & 3635.00 & -0.59 & .55 & .66 \\
Movement & 3816.00 & 0.89 & .38 & .38 \\
Feeding & 3880.50 & 1.17 & .24 & .24 \\
Maintenance & 3674.50 & -0.08 & .93 & .93 \\
Inactivity & $\mathbf{3 2 5 5 . 0 0}$ & $\mathbf{- 2 . 2 4}$ & $\mathbf{. 0 3}$ & $\mathbf{. 0 3}$ \\
Social Proximity & $\mathbf{2 5 3 3 . 0 0}$ & $\mathbf{- 5 . 1 4}$ & $<.001$ & $\mathbf{. 0 0 1}$ \\
Path Proximity & 3591.00 & -0.86 & .39 & .41 \\
\hline
\end{tabular}

Note. Significant $(p<.05)$ results are highlighted in bold.

Figure 2

Percent \pm SE of Visible Kangaroos Engaged in Each Behavior Between Zoo Status Conditions

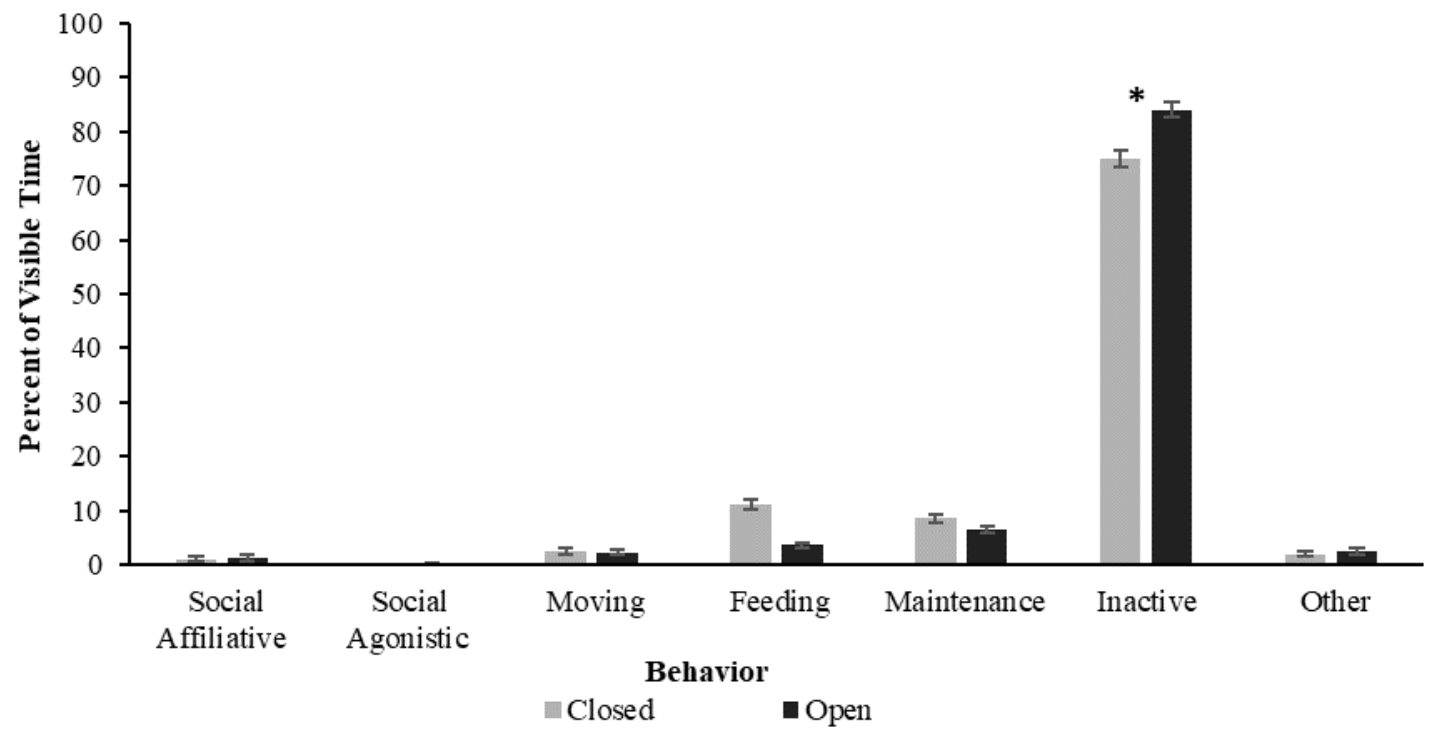

Note. Significant $(p<.05)$ results are marked with an asterisk.

Our space use analyses revealed that space use was significantly influenced by zoo status $(F(1$, $5)=83.99, p<.001$ ) (Figure 3). Specifically, space use was higher when the zoo was closed than when the zoo was open, regardless of the time of day. We also found a significant interaction between time of day and zoo status such that, when the zoo was open, space use was higher in the morning hours than in the midday or afternoon hours $(F(2,5)=6.49, p=.002)$. There was no significant difference in space use with time of day when the zoo was closed. However, aside from inactivity, social proximity, and space use, no other tested dependent variable was significantly influenced by zoo status (Table 3 ). 
Jones et al. 529

Figure 3

Variation in Space Use between Zoo Status Conditions, Where the SE Represents the Variation Between Individuals

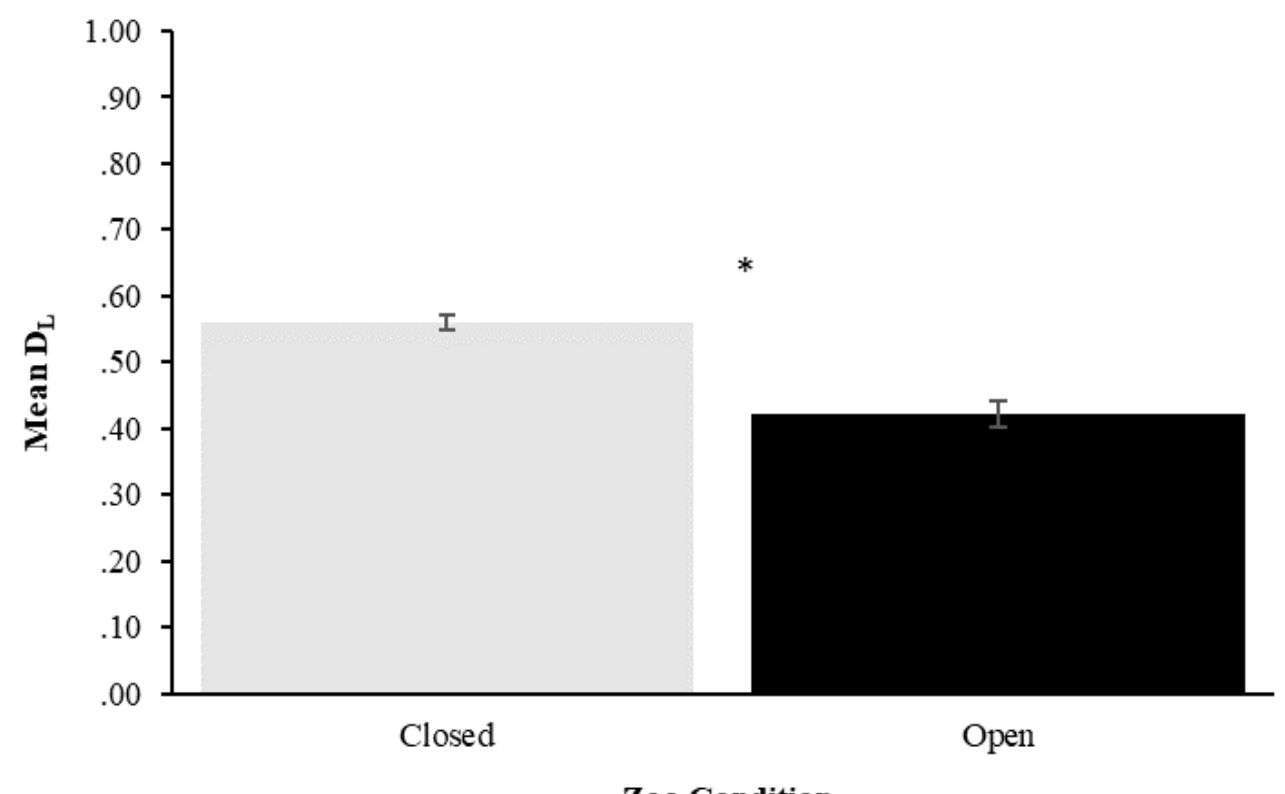

Note. Significant $(p<.05)$ results are marked with an asterisk.

\section{Crowd Size}

Analyses of the impact of crowd size showed significant variation for feeding behavior (Table 4). Feeding was highest when no visitors were present $\left(10.11^{\wedge} \pm 2.17 \%, N=65\right)$ and in low crowds of 1-5 visitors $(9.44 \% \pm 3.11 \%, N=16)$ as compared to crowds of $6-10$ visitors $(0.60 \% \pm 0.60 \%, N=12), 11-$ 15 visitors $(1.14 \% \pm 0.62 \%, N=18)$ or crowds of $16+$ visitors $(1.86 \% \pm 1.32 \%, N=11)$ (Table 4$)$. All individuals were observed feeding when no visitors were present, with feeding accounting for a range of between $7.55 \% \pm 0.24 \%$ (Stirling) to $16.67 \% \pm 0.35 \%$ (Zora) of total visible time during this crowd condition. Some individuals including Kelly, Logan, Mackay, Missy, and Stirling were never observed feeding when visitors of any quantity were present (Figure 4). Hampton, Isa, Jasper, Kiki, Lilly, and Marrabel all displayed an increase in percent of visible time spent feeding between when no visitors were present and crowd sizes of one to five visitors. No individual was observed feeding during every crowd size condition (Figure 4). These crowd size effects may potentially be explained by confounding environmental variables. We found that feeding was significantly negatively correlated with time of day $(r(120)=-.30, p<.001)$ such that feeding decreased from morning to afternoon. We also found that feeding was negatively correlated with temperature $(r(120)=-.21, p=.02)$.

In terms of crowd sizes, we observed the opposite trend for social proximity (Figure 5). Social proximity was lowest when no visitors were present $(59.30 \% \pm 2.89 \%, N=63)$, and higher in crowds of $1-5(80.34 \% \pm 3.21 \%, \mathrm{~N}=16), 6-10(81.26 \% \pm 3.57 \%, N=12), 11-15(83.62 \% \pm 2.80 \%, N=18)$, or $16+$ visitors $(85.06 \% \pm 3.56 \%, N=11)$. Time spent in social proximity increased with crowd size across several individuals including Grafton, Hampton, Isa, Jasper, Stirling, and Rae (Figure 5). This was particularly true for Boomer, Grafton, and Jasper that spent $100 \%$ of their time in social proximity with another individual during crowd sizes of either 11-15 visitors or 16+ visitors (Figure 5).

We also found that space use was significantly influenced by crowd size $(F(4,70)=33.96, p<$ .001) such that space use decreased as crowd size increased. However, aside from feeding, social 
proximity, and space use, no other tested dependent variable was significantly influenced by crowd size (Figure 5).

No tested dependent variable was significantly correlated with weather. Neither social affiliation, maintenance, nor path proximity were significantly correlated with any tested condition or environmental variables. However, we did find a significant negative correlation between movement and time of day $(r$ $(120)=-.20, p=.03)$ such that movement decreased from morning to afternoon. This may partially explain the observed relationship between space use and time of day $(F(2,5)=12.07, p<.001)$, which demonstrated that space use was the greatest during the morning hours under both zoo status conditions.

Table 4

Kruskal-Wallis Tests with Monte Carlo Correction Results for Behavioral Variation Between Crowd Size Conditions

\begin{tabular}{lcccc}
\hline \multicolumn{1}{c}{ Behavior } & $X^{2}$ & DF & $\operatorname{Pr}>X^{2}$ & $\operatorname{Pr} \geq X^{2}$ \\
\hline Social Affiliation & 4.05 & 4 & .40 & .38 \\
Movement & 2.59 & 5 & .63 & .64 \\
Feeding & $\mathbf{1 2 . 4 7}$ & $\mathbf{4}$ & $\mathbf{. 0 1}$ & $\mathbf{. 0 1}$ \\
Maintenance & 2.56 & 5 & .63 & .64 \\
Inactivity & 7.30 & 5 & .12 & .12 \\
Social Proximity & $\mathbf{3 3 . 4 9}$ & $\mathbf{4}$ & $<.001$ & $\mathbf{. 0 0 1}$ \\
Path Proximity & 3.52 & 4 & .48 & .49 \\
\hline
\end{tabular}

Note. Significant $(p<.05)$ results are highlighted in bold.

\section{Figure 4}

Percent \pm SE of Visible Time Individuals Spent Feeding Across Crowd Size Conditions

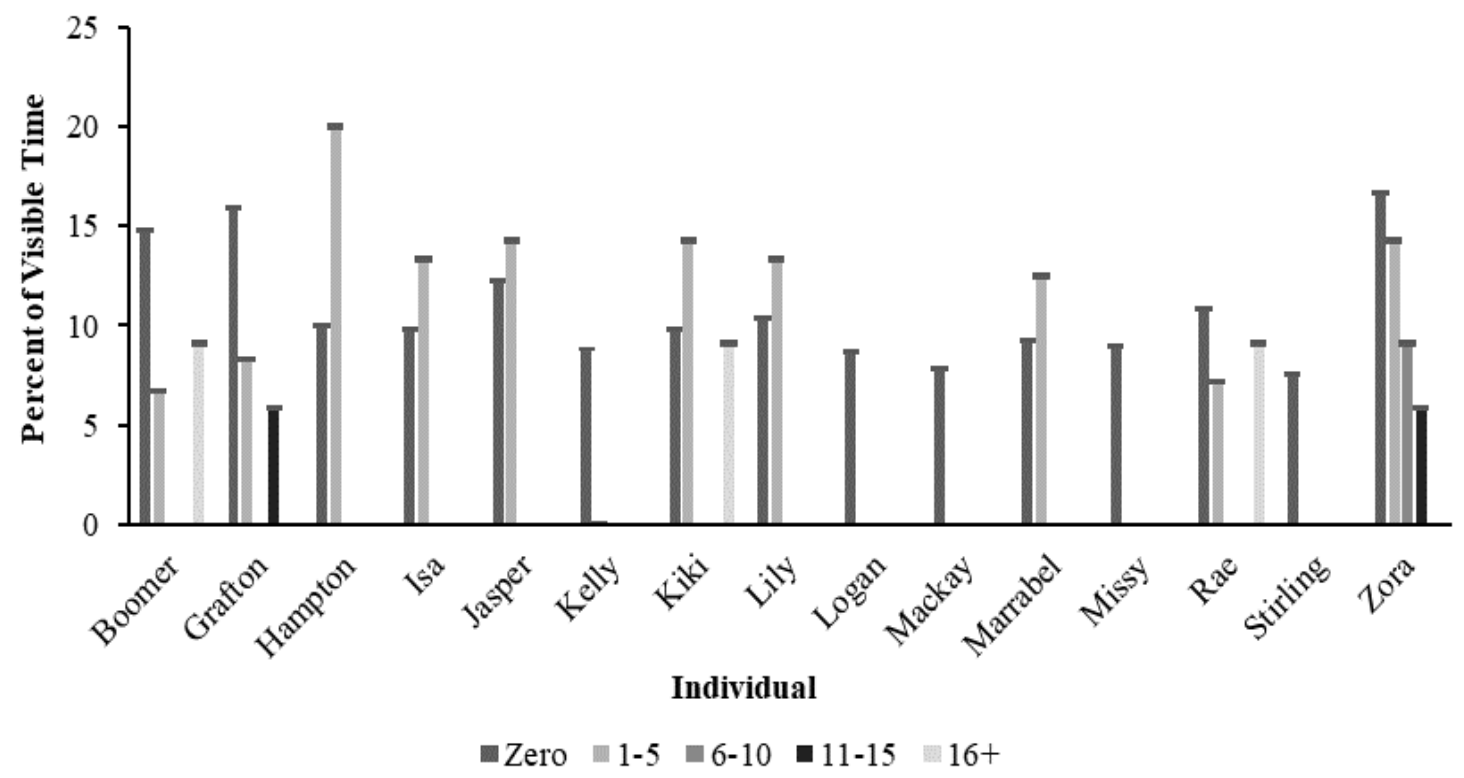


Figure 5

Percent \pm SE of Visible Time Individuals Spent in Social Proximity Across Crowd Size Conditions

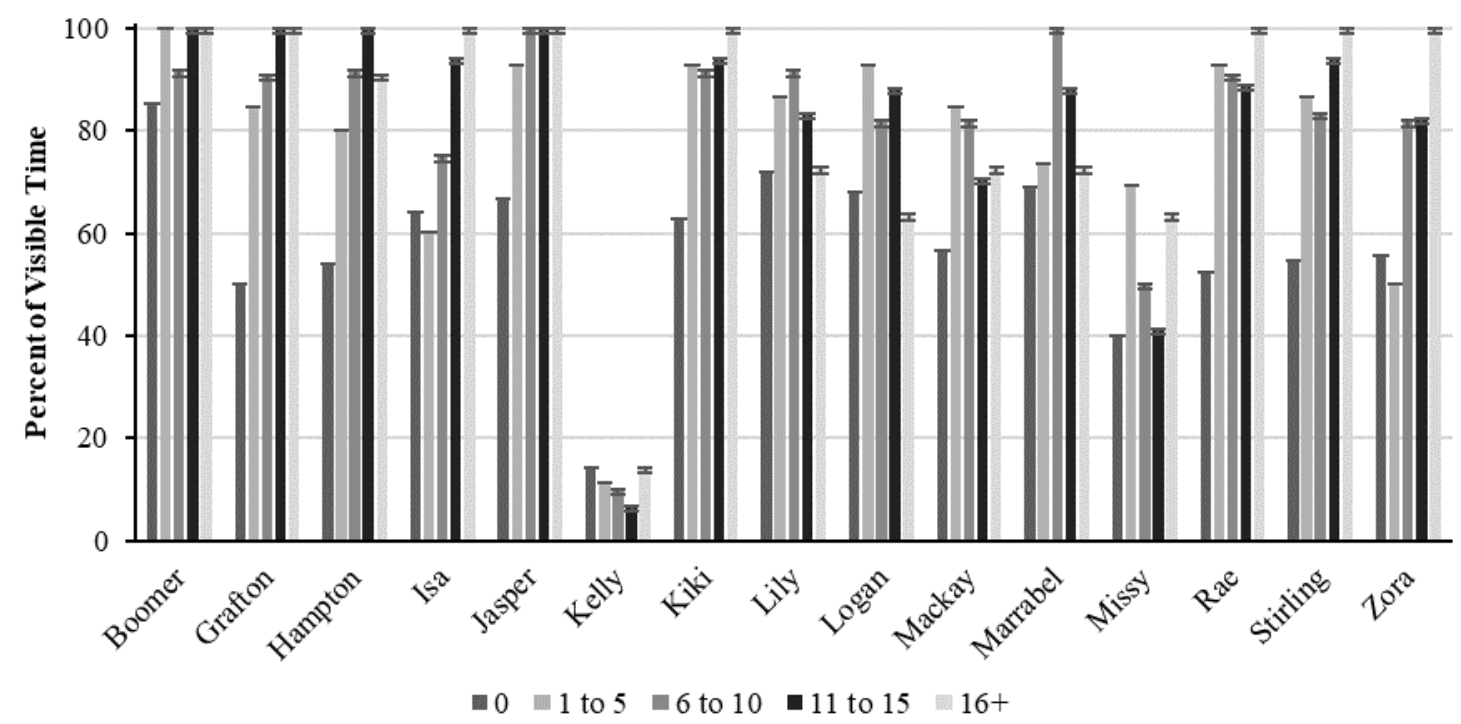

Note. The zero crowd size condition includes data from both zoo status conditions (i.e., open and closed) in order to maintain evenness of sample sizes for each crowd size condition.

\section{Discussion}

\section{Zoo Status}

We expected zoo status to significantly impact behavior and space use following observations in other species (Blanchett et al., 2020; Learmonth et al., 2018; Lockley \& Leadbeater, 2005). Inactivity and social proximity were significantly higher when the zoo reopened with limited guest capacity. The increase in social proximity when the zoo reopened may have been partially due to temperature or aggregation in an area of the habitat preferred by the kangaroos, but it may also be related to their natural anti-predator grouping behaviors (Blumstein \& Daniel, 2003; Frith \& Calaby, 1969).

Regardless of time of day, space use was higher when the zoo was closed than when the zoo was open, suggesting a potential visitor impact. When the zoo was open, space use was highest in the morning, when there were typically fewer visitors present as compared to either midday or afternoon hours. Similar results have been reported in parma wallabies (Macropus parma) in walk-through habitats (Rendle et al., 2018). Space use analyses demonstrated that these kangaroos primarily congregated under a mature tree near the public entrance regardless of zoo status. Although there were alternate sources of shade, congregating in the source of shade nearest to the public entrance may reflect a group anti-predator strategy to avoid being surprised by visitors. In a study by Banks (2001), kangaroos changed how far away they foraged from refuge based on predation risk, so that they spent more time foraging closer to refuge when predation risk was high. The location the Detroit Zoo kangaroos preferred near the visitor entrance was also near one of the structures the kangaroos often utilized to shelter from rain, although there are multiple refuges further away from the visitor entrance. However, because kangaroos are grassland and desert animals, they rely on group vigilance as one of their primary anti-predator strategies (Blumstein \& Daniel, 2003; Frith \& Calaby, 1969), which may be a factor in why the kangaroos at the Detroit Zoo might prefer to use a location where visitors are clearly visible.

Only a handful of visitor effect studies, none of which included red kangaroos, have been published where animals were subjected to prolonged periods of time without visitors. Grevy's zebras 
(Equus grevyi) reportedly spent significantly more time closer to public viewing areas when a zoo was closed due to the COVID-19 pandemic (Williams et al., 2021a), and slender-tailed meerkats (Suricata suricatta) used more of their habitat during the prolonged period without visitors (Williams et al., 2021b). In contrast, African penguins (Spheniscus demersus) showed no changes in behavior or space use between zoo status conditions (Williams et al., 2021b). These species all have vastly different natural histories and therefore are likely to perceive threats very differently (Queiroz \& Young, 2018), highlighting the need for more research on visitor effects in order to more accurately predict which zoohoused species will likely need habitat modifications to promote good welfare.

\section{Crowd Size}

Crowd size had a significant impact on a number of group-level behaviors. However, the zoo reopened under substantially reduced attendance capacity, and the effects of crowd size we measured may not be representative of normal conditions. Although Meade et al. (2021) found that crowd size significantly impacted resting behavior in two species of wallaby, the same trend did not apply to red kangaroos and was not observed in this study at the reduced visitor capacity. However, Sherwen Hemsworth, et al. (2015) reported that red kangaroos decreased inactivity with higher visitor numbers, and both studies found that red kangaroos spent more time displaying visitor-directed vigilance in higher crowd sizes (Meade et al., 2021; Sherwen, Hemsworth, et al., 2015). When drawing comparisons to these studies we must consider that we did not record vigilance behavior, so rates of inactivity are likely skewed between studies.

Despite the limitations on crowd size, the kangaroos displayed significantly higher rates of feeding when guests were not present than in higher crowd size conditions. This result is inconsistent with two other visitor effect studies on red kangaroos, both of which found that foraging behavior was not affected by crowd size (Meade et al., 2021; Sherwen, Hemsworth et al., 2015). However, we also found that feeding was negatively correlated with time of day such that feeding decreased from morning to afternoon. Since kangaroos are largely nocturnal and typically start their daily foraging at dusk and continue into dawn, this finding is consistent with the species natural history (Frith \& Calaby, 1969; Strahan, 1983; Wilson \& Mittermeier, 2015).

The kangaroos spent less time in proximity to each other and had a greater spatial distribution in the habitat when there were no visitors compared to all other crowd size conditions. This suggests an impact of visitor presence and density on social proximity and space use patterns, providing support that the differences we saw based on zoo status were related to visitors rather than potential seasonal confounds. Interestingly, there was a slight increase in space use in crowds of 16+, which may serve an unidentified strategic purpose. Blanchett et al. (2020) found that birds living in free-flight aviaries appeared to use their space in ways that mitigated any potential negative impacts of high visitor numbers. It is possible that there may be an advantage to spatial distributions during peak crowd size conditions that may not be necessary during smaller crowd size conditions. Further studies are necessary to clarify the existence and function of spatial distribution as a high crowd mitigation strategy.

\section{Potential Environmental Confounds}

Previous studies on visitor effects have cautioned against overestimating the impacts of natural fluctuations in visitors (Goodenough et al., 2019) by considering that weather and time of day often covary with visitor numbers (Álvarez \& Barquín, 2020; Aylen et al., 2014; Hewer \& Gough, 2016; Sherwen \& Hemsworth, 2019; Su \& Lin, 2019). The onset of COVID-19 defined the temporal limitations of our study in March of 2020. As such, zoo closure data were collected in the spring, while reopening data were collected in the summer, which prevented reasonable control of environmental variables. We tested temperature and weather condition effects and found a few significant results in relation to temperature. We saw increased inactivity during higher temperature conditions, which was unsurprising given that kangaroos typically spend most of their time resting and searching for shade during the hottest parts of the 
day (Frith \& Calaby, 1969; Strahan, 1983; Wilson \& Mittermeier, 2015). Interestingly, social proximity was also positively correlated with temperature so that individuals spent more time in proximity to each other as temperatures increased. This relationship contradicts typical thermoregulatory strategies where animals group together as temperatures decrease (Breed \& Moore, 2015; Cheleuitte-Nieves et al., 2020). This, in combination with the observed increase in social density with increased crowd size, supports our hypothesis that shifts in social proximity are influenced by visitor effects rather than temperature.

\section{Implications for Animal Welfare}

Our results coincided with our predictions based on the Queiroz and Young (2018) model, from which we identified several aspects of red kangaroo natural history that may have influenced their reaction to visitors. The model predicts that kangaroos, as a terrestrial species, should be impacted by visitors more than arboreal species. Additionally, visitors typically have less impact on nocturnal species like kangaroos as they are often inactive during visiting hours. Finally, as a species adapted to open habitats (i.e., grasslands), the model predicts that kangaroos should experience fewer visitor effects than species accustomed to hiding from predators. The mixed results from this study are thus consistent with the moderate impact of visitors on red kangaroos predicted by the model.

Changes in activity budgets can be difficult to interpret and do not necessarily mean that animal welfare has changed (Watters et al., 2021). Increased social proximity could function as a form of vigilance behavior, but the relationship between vigilance and animal welfare can be ambiguous. Some studies have interpreted vigilance as curiosity, meaning the behavior fits relatively neutrally in the scope of animal welfare (Sherwen, Hemsworth et al., 2015). Others have interpreted vigilance as fear, which may have deleterious effects on welfare (Birke, 2002; Carder \& Semple, 2008). Other behaviors typically included in activity budgets, such as inactivity or locomotion, are not necessarily indicative of welfare states (Davey, 2007; Sherwen \& Hemsworth, 2019). Animals naturally display shifts in behavior according to environmental stimuli, which may help mitigate any potential negative impacts of high visitor numbers (Blanchett et al., 2020). Although behavior changes are helpful indicators of visitor effects, how these changes impact overall welfare remains in question (Davey, 2007). Although not widely described in the literature, potential welfare indicators for kangaroos include excessive forearm licking and tintibulation (i.e., upper body trembling). However, these behaviors have limitations, as forearm licking is also a form of thermoregulation, and tintibulation can be expressed either as excitement or anxiety (Vogelnest, 2015).

In the absence of clear indicators of welfare, a comprehensive welfare evaluation should include not only measurements of behavior but also physiological and emotional measures. Emotions are relatively subjective states that have been proven difficult to measure in animals (Hemsworth et al., 2015), and studies like ours are limited in collecting physiological data by the challenges of non-invasive data collection amongst large groups. To more thoroughly evaluate the effects of visitors, we chose to incorporate space use into our behavioral monitoring. Although the kangaroos had an expansive 7,896 $\mathrm{m}^{2}$ habitat, they primarily used a smaller section of the habitat near the visitor entrance during the study. Animals that concentrate their space use within a habitat may also be behaviorally restricted due to limited choices in the area of the habitat they are comfortable using, emphasizing the importance of considering elements of habitat design in visitor effect studies, particularly for walk-through habitats. Habitat features designed to limit human-animal interaction have been known to reduce stress in various species (Fernandez et al., 2009). Habitat design did affect feeding behavior in a study of Bennett's wallabies (Macropus rufogriseus), such that animals in habitats where contact was prohibited spent more time feeding than those in habitats allowing for visitor contact (Beaudin-Judd et al., 2019). Further research should be conducted to explore how visitors might impact the usage of habitat features, allowing zoos to implement designs that may lessen the effects of visitors.

According to the human-animal relationship model by Hosey (2008), predicting an animal's relationship to humans is first based on the species-specific fear of humans. Red kangaroos in the wild are frequently found in close proximity to humans (Wilson \& Mittermeier, 2015), suggesting they are 
relatively impartial to the presence of humans. The next stage in the model considers an animal's interactions with both familiar and unfamiliar humans. Here we must consider that most of our study subjects were born and raised in their current habitat at the Detroit Zoo and are likely habituated to the presence of visitors. Based on the model, these factors likely contributed to the kangaroos' relatively minimal reactions towards visitors even after a significant period without them present.

To ensure the needs of individuals are being met, it is important for studies on group-housed animals to also monitor the behavior of individuals. Our individual monitoring results revealed that Grafton, Hampton, Isa, Jasper, Stirling, and Rae all exhibited an increase in social proximity when the zoo was open, suggesting that these individuals may find additional comfort within a group setting. Kelly, an individual that was rescued from a private owner, spent significantly less time in social proximity than any other individual, regardless of zoo status or crowd size. After living as a pet, Kelly likely did not have the appropriate social skills to effectively integrate into group life upon his arrival at the Detroit Zoo. Missy, the eldest kangaroo in the group, was also an outlier in terms of social proximity. Although these particular individual differences can be mitigated by the animals themselves through movement and dispersal (with the caveat of having an appropriately sized habitat), other trends in individual behavior may be alleviated through their management. For example, a number of individuals including Kelly, Logan, Mackay, Missy, and Stirling were never observed feeding when visitors of any quantity were present. This finding validates efforts to mitigate visitor impact, such as accessible indoor feeding areas out of view of guests. It is possible these individuals did feed during these times in their indoor habitat, which was not visible to observers. By taking individual measurements of behavior and space use, animal care staff can make informed decisions that are tailored to the needs of individuals.

\section{Conclusion}

Although this opportunistic study of visitor effects was able to capture data from an extended period without visitors, it was limited by seasonal confounds and substantially reduced visitor capacity, which presented difficulties in accurate measurement of true visitor impacts. Our data revealed a number of differences in behavior that coincided with kangaroo natural history, but others that could be evidence for potential visitor effects. Overall, we noticed similar trends across zoo status and crowd size conditions that provided evidence that use of space and social proximity were based more on visitors than environmental confounds. Although our data suggested that kangaroos changed their behavior and space use in response to visitors, the implications for their welfare remain unclear. Further research is needed in order to validate indicators of welfare for this species.

\section{Acknowledgements}

We thank the animal care staff at the Detroit Zoo for their support of this project, and three anonymous reviewers for their helpful feedback on earlier versions of this manuscript.

\section{References}

Altmann, J. (1974). Observational study of behavior: Sampling methods. Behaviour, 49(3-4), 227-266.

Aylen, J., Albertson, K., \& Cavan, G. (2014). The impact of weather and climate on tourist demand: The case of Chester Zoo. Climatic Change, 127(2), 183-197.

Banks, P. B. (2001). Predation-sensitive grouping and habitat use by eastern grey kangaroos: A field experiment. Animal Behaviour, 61(5), 1013-1021.

Beaudin-Judd, J., Weladji, R. B., Lazure, L., \& Paré, P. (2019). Activity budget and spatial distribution of Bennett's wallabies (Macropus rufogriseus) in open versus closed exhibit designs. Zoo Biology, 38(3), 258-265.

Belton, L. E., Cameron, E. Z., \& Dalerum, F. (2016). Spotted hyaena space use in relation to human infrastructure inside a protected area. PeerJ, 4, e2596. 
Blanchett, M. K. S., Finegan, E., \& Atkinson, J. (2020). The effects of increasing visitor and noise levels on birds within a free-flight aviary examined through enclosure use and behavior. Animal Behavior and Cognition, 7(1), 49-69.

Blumstein, D. T., \& Daniel, J. C. (2003). Red kangaroos (Macropus rufus) receive an antipredator benefit from aggregation. Acta Ethologica, 5(2), 95-99.

Bonnie, K. E., Ang, M. Y., \& Ross, S. R. (2016). Effects of crowd size on exhibit use by and behavior of chimpanzees (Pan troglodytes) and Western lowland gorillas (Gorilla gorilla) at a zoo. Applied Animal Behaviour Science, 178, 102-110.

Boyle, S. A., Berry, N., Cayton, J., Ferguson, S., Gilgan, A., Khan, A., Lam, H., Leavelle, S., Mulder, I., Myers, R., Owens, A., Park, J., Siddiq, I., Slevin, M., Weidow, T., Yu, A. J., \& Reichling, S. (2020). Widespread behavioral responses by mammals and fish to zoo visitors highlight differences between individual animals. Animals, 10(11), 2108.

Breed, M. D., \& Moore, J. (2015). Animal behavior. Academic Press.

Brereton, J. E. (2020). Directions in animal enclosure use studies. Journal of Zoo and Aquarium Research, 8(1), 1-9.

Carder, G., \& Semple, S. (2008). Visitor effects on anxiety in two captive groups of western lowland gorillas. Applied Animal Behaviour Science, 115(3-4), 211-220.

Chamove, A. S., Hosey, G. R., \& Schaetzel, P. (1988). Visitors excite primates in zoos. Zoo Biology, 7(4), 359-369.

Cheleuitte-Nieves, C., Perotto-Baldivieso, H. L., Wu, X. B., \& Cooper, S. M. (2020). Environmental and landscape influences on the spatial and temporal distribution of a cattle herd in a South Texas rangeland. Ecological Processes, 9(1), 1-18.

Chiapero, F., Ferrari, H. R., Prieto, M. V., Garcia Capocasa, M. C., \& Busso, J. M. (2021). Multivariate analyses of the activity pattern and behavior of the lesser anteater on open and closed days at Córdoba Zoo, Argentina. Journal of Applied Animal Welfare Science, 24(1), 83-97.

Chiew, S. J., Butler, K. L., Sherwen, S. L., Coleman, G. J., Fanson, K. V., \& Hemsworth, P. H. (2019). Effects of regulating visitor viewing proximity and the intensity of visitor behaviour on little penguin (Eudyptula minor) behaviour and welfare. Animals, 9(6), 285.

Chiew, S. J., Butler, K. L., Sherwen, S. L., Coleman, G. J., Melfi, V., Burns, A., \& Hemsworth, P. H. (2020). Effect of covering a visitor viewing area window on the behaviour of zoo-housed little penguins (Eudyptula minor). Animals, 10(7), 1224.

Colgrave, N.J., Engel, J., \& Plowman, A. B., (2006). Randomization tests. In A.B. Plowman (Ed.), Zoo research guidelines: Statistics for typical zoo datasets (pp. 7-16). BIAZA.

Davey, G. (2007). Visitors' effects on the welfare of animals in the zoo: A review. Journal of Applied Animal Welfare Science, 10(2), 169-183.

Domico, T. (1993). Kangaroos: The marvelous mob. Facts on File.

Eltorai, A. E., \& Sussman, R. W. (2010). The "visitor effect" and captive black-tailed prairie dog behavior. Der Zoologische Garten, 79(2-3), 109-120.

Fernandez, E. J., Tamborski, M. A., Pickens, S. R., \& Timberlake, W. (2009). Animal-visitor interactions in the modern zoo: Conflicts and interventions. Applied Animal Behaviour Science, 120(1-2), 1-8.

Frith, H. J., \& Calaby, J. H. (1969). Kangaroos. Cheshire.

Goodenough, A. E., McDonald, K., Moody, K., \& Wheeler, C. (2019). Are" visitor effects" overestimated? Behaviour in captive lemurs is mainly driven by co-variation with time and weather. Journal of Zoo and Aquarium Research, 7(2), 59-66.

Halliday, T. R. (1983). Information and communication. In P. J. B. Slater \& T. R. Halliday (Eds.), Animal behaviour: Communication (pp. 43-81). W. H. Freeman and Company

Hashmi, A., \& Sullivan, M. (2020). The visitor effect in zoo-housed apes: The variable effect on behaviour of visitor number and noise. Journal of Zoo and Aquarium Research, 8(4), 268-282.

Hemsworth, P. H., Mellor, D. J., Cronin, G. M., \& Tilbrook, A. J. (2015). Scientific assessment of animal welfare. New Zealand Veterinary Journal, 63(1), 24-30.

Hosey, G. R. (2000). Zoo animals and their human audiences: What is the visitor effect? Animal Welfare, 9(4), 343357.

Hosey, G. (2008). A preliminary model of human-animal relationships in the zoo. Applied Animal Behaviour Science, 109(2-4), 105-127.

Kagan, R., Carter, S., \& Allard, S. (2015). A universal animal welfare framework for zoos. Journal of Applied Animal Welfare Science, 18, S1-S10. 
Kelly, K. R., Harrison, M. L., Size, D. D., \& MacDonald, S. E. (2015). Individual effects of seasonal changes, visitor density, and concurrent bear behavior on stereotypical behaviors in captive polar bears (Ursus maritimus). Journal of Applied Animal Welfare Science, 18(1), 17-31.

Larsen, M. J., Sherwen, S. L., \& Rault, J. L. (2014). Number of nearby visitors and noise level affect vigilance in captive koalas. Applied Animal Behaviour Science, 154, 76-82.

Learmonth, M. J., Sherwen, S., \& Hemsworth, P. H. (2018). The effects of zoo visitors on quokka (Setonix brachyurus) avoidance behavior in a walk-through exhibit. Zoo Biology, 37(4), 223-228.

Leeds, A., \& Lukas, K. E. (2019). Monitoring the social behavior of a bachelor mandrill (Mandrillus sphinx) dyad participating in touchscreen-mediated cognitive testing. Zoo Biology, 38(4), 397-402.

Lockley, D., \& Leadbeater, J. (2005, July). The effect of visitor impacts on behavioural repertoires and use of enclosure captive red-necked wallabies (Macropus rufogriseus fruticus) at Chester Zoological Gardens. In Proceedings of the 7th Annual Symposium on Zoo Research, Twycross Zoo, Warwickshire, UK (pp. 238-247). British and Irish Association of Zoos and Aquariums.

Mallapur, A., \& Chellam, R. (2002). Environmental influences on stereotypy and the activity budget of Indian leopards (Panthera pardus) in four zoos in Southern India. Zoo Biology, 21(6), 585-595.

Mallapur, A., Sinha, A., \& Waran, N. (2005). Influence of visitor presence on the behaviour of captive lion-tailed macaques (Macaca silenus) housed in Indian zoos. Applied Animal Behaviour Science, 94(3-4), 341-352.

Margulis, S. W., Hoyos, C., \& Anderson, M. (2003). Effect of felid activity on zoo visitor interest. Zoo Biology, 22(6), 587-599.

Meade, J., Melfi, V. A., Keith, M., \& Burns, A. L. (2021). The effect of visitor number on the behavior of zoohoused macropods. Anthrozoös, 34(4), 1-13.

Mellor, D. J., \& Beausoleil, N. J. (2015). Extending the 'Five Domains' model for animal welfare assessment to incorporate positive welfare states. Animal Welfare, 24(3), 241-253.

Mellor, D. J., Beausoleil, N. J., Littlewood, K. E., McLean, A. N., McGreevy, P. D., Jones, B., \& Wilkins, C. (2020). The 2020 five domains model: Including human-animal interactions in assessments of animal welfare. Animals, 10(10), 1870.

Mellor, D. J., \& Reid, C. S. W. (1994). Concepts of animal well-being and predicting the impact of procedures on experimental animals. In Improving the well-being of animals in the research environment; Australian and New Zealand Council for the Care of Animals in Research and Teaching (pp. 3-18).

Mittermeier, R. A., Rylands, A. B., \& Wilson, D. E. (2013). Handbook of mammals of the world, Vol 3. Primates. Lynx Edicions.

O'Donovan, D., Hindle, J. E., McKeown, \& O'Donovan, S. (1993). Effect of visitors on the behaviour of female cheetahs Acinonyx jubutus and cubs. International Zoo Yearbook, 32(1), 238-244

Payne, L. X., Schindler, D. E., Parrish, J. K., \& Temple, S. A. (2005). Quantifying spatial pattern with evenness indices. Ecological Applications, 15(2), 507-520.

Plowman, A. B. (2008). BIAZA statistics guidelines: Toward a common application of statistical tests for zoo research. Zoo Biology, 27, 226-233.

Queiroz, M. B., \& Young, R. J. (2018). The different physical and behavioural characteristics of zoo mammals that influence their response to visitors. Animals, 8(8), 139.

Rendle, J. A. J., Ward, S., \& McCormick, W. D. (2018). Behaviour and enclosure use of captive parma wallabies (Macropus parma): An assessment of compatibility within a mixed-species exhibit. Journal of Zoo and Aquarium Research, 6(2), 63-68.

Rose, P. E., Brereton, J. E., \& Croft, D. P. (2018). Measuring welfare in captive flamingos: Activity patterns and exhibit usage in zoo-housed birds. Applied Animal Behaviour Science, 205, 115-125

Ross, M. R., Niemann, T., Wark, J. D., Heintz, M. R., Horrigan, A., Cronin, K. A., Shender, M. A., Gillespie, K. (2016). ZooMonitor (Version 3) [Mobile app]. http://zoomonitor.org.

Sellinger, R. L., \& Ha, J. C. (2005). The effects of visitor density and intensity on the behavior of two captive jaguars (Panthera onca). Journal of Applied Animal Welfare Science, 8(4), 233-244.

Shannon, C. E., Weaver, W. (1948). The mathematical theory of communication. University of Illinois Press.

Sherwen, S. L., Harvey, T. J., Magrath, M. J., Butler, K. L., Fanson, K. V., \& Hemsworth, P. H. (2015). Effects of visual contact with zoo visitors on black-capped capuchin welfare. Applied Animal Behaviour Science, 167, 65-73.

Sherwen, S. L., \& Hemsworth, P. H. (2019). The visitor effect on zoo animals: Implications and opportunities for zoo animal welfare. Animals, 9(6), 366.

Sherwen, S. L., Hemsworth, P. H., Butler, K. L., Fanson, K. V., \& Magrath, M. J. (2015). Impacts of visitor number on kangaroos housed in free-range exhibits. Zoo Biology, 34(4), 287-295. 
Sherwen, S. L., Magrath, M. J., Butler, K. L., \& Hemsworth, P. H. (2015). Little penguins, Eudyptula minor, show increased avoidance, aggression and vigilance in response to zoo visitors. Applied Animal Behaviour Science, 168, 71-76.

Stevens, J., Thyssen, A., Laevens, H., \& Vervaecke, H. (2013). The influence of zoo visitor numbers on the behaviour of harbour seals (Phoca vitulina). Journal of Zoo and Aquarium Research, 1(1), 31-34.

Stoinski, T. S., Jaicks, H. F., \& Drayton, L. A. (2012). Visitor effects on the behavior of captive western lowland gorillas: The importance of individual differences in examining welfare. Zoo Biology, 31(5), 586-599.

Strahan, R. (1983). Complete book of Australian mammals. Angus \& Robertson.

Suárez, P., Recuerda, P., \& Arias-de-Reyna, L. (2017). Behaviour and welfare: The visitor effect in captive felids. Animal Welfare, 26(1), 25-34.

Todd, P. A., Macdonald, C., \& Coleman, D. (2007). Visitor-associated variation in captive Diana monkey (Cercopithecus diana diana) behaviour. Applied Animal Behaviour Science, 107(1-2), 162-165.

Vogelnest, L. (2015). Marsupialia (marsupials). Fowler's Zoo and wild Animal Medicine, 8, 255-274.

Watters, J. V., Krebs, B. L., \& Eschmann, C. L. (2021). Assessing animal welfare with behavior: Onward with caution. Journal of Zoological and Botanical Gardens, 2(1), 75-87.

Webster, J. (1995). Animal welfare: a cool eye towards Eden. Blackwell Science Ltd. Wells, D. L. (2005). A note on the influence of visitors on the behaviour and welfare of zoo-housed gorillas. Applied Animal Behaviour Science, 93(1-2), 13-17.

Williams, E., Carter, A., Rendle, J., \& Ward, S. J. (2021a). Impacts of COVID-19 on animals in zoos: A longitudinal multi-species analysis. Journal of Zoological and Botanical Gardens, 2(2), 130-145.

Williams, E., Carter, A., Rendle, J., \& Ward, S. J. (2021b). Understanding impacts of zoo visitors: Quantifying behavioural changes of two popular zoo species during COVID-19 closures. Applied Animal Behaviour Science, 236, 105253.

Wilson, D. E., Mittermeier, R. A. (2015). Handbook of mammals of the world, Vol 5. Monotremes and Marsupials. Lynx Edicions. 